\title{
Sexual risk behaviour and infection: epidemiological considerations
}

\section{SO Aral}

$\mathrm{R}$ esearchers into sexually transmitted disease (STD) have been trying to measure sexual behaviour for a very long time. Following the emergence of the HIV pandemic in the 1980s and 1990s, the focus on sexual measurement intensified. Researchers attempted to measure sexual behaviour in a wide variety of contexts for various purposes, often without explicit attention to the impact of context and purpose on measurement. ${ }^{1-15}$ Investigators measured sexual behaviour in STD and family planning clinics to assess clients' risk for sexually transmitted infection (STI): in infected and uninfected populations to identify behavioural risk factors for STI; in specific groups to describe and identify high and low risk subpopulations; in general populations to describe behaviour patterns and monitor changing trends through time, and finally in intervention trials and intervention programmes to assess efficacy and effectiveness of behavioural interventions. Often the same sexual behaviour questions were used for different purposes and in the variety of contexts mentioned above.

The accumulating experience in sexual behaviour measurement, as it relates to STDs, suggests that the "one size fits all" approach may be inadequate for the diverse purposes at hand. A careful analysis of what needs to be measured for different purposes, in different contexts, may be needed to help move the sexual behaviour measurement field forward.

This paper describes the measurement of sexual behaviour in the context of STI transmission. It reviews studies undertaken (1) to monitor sexual behaviour and (2) to assess the role of specific sexual behaviours in the transmission of specific STIs. Issues discussed include:

- the distribution of STIs and high risk sexual behaviour in populations;

- the role of core groups in STD transmission dynamics and their implications for the measurement of sexual behaviour;

- the measurement of temporal changes in sexual behaviour and the relation between timing of sexual behaviour measurement and STI/STD prevalence and incidence;

- the importance of context in sexual behaviour measurement and the interdependencies among sexual behaviours that need to be considered in interpreting the behaviours being measured;

- the role of study populations in the measurement of behaviours related to the transmission probability, duration of infectiousness, and rate of sexual contact between infected and uninfected people, for specific sexually transmitted pathogens.

Consideration of these issues may shed some light on the specific behaviours to be measured, the time interval over which they should be measured, and the populations in which measurement should take place.

\section{MONITORING SEXUAL BEHAVIOUR}

It is remarkable that, despite the great variation in both morbidities and risk behaviours, a systematic approach to the assessment and interpretation of the distribution of sexual behaviours in populations has not yet been developed. Measures of central tendency still seem to be the sexual behaviour indicators of choice. The accumulating data on HIV, other STDs, and sexual behaviour all point to their vast variability in populations. One recent analysis of HIV prevalence and incidence found wide variations in categories of exposure even across countries which have the same type of epidemic. ${ }^{16}$ Data on number of sex partners, from developed and developing countries, indicate that the majority of people in a population have few sex partners while a small minority report very large numbers. ${ }^{17-19}$ Similarly, data on frequency of sexual intercourse and sexual practices reveal great variation even, for example, within the population of a small US city. ${ }^{20}$

\section{Core groups}

Monitoring the sexual behaviour of so called "core groups" is important in the spread and prevention of STI. ${ }^{21-23}$ Core groups are defined as "small proportions of persons with an STD who are frequently infected with and transmit the disease, and who sustain the endemic and epidemic transmission of STD". ${ }^{21}$ Recent work on modelling of dynamic and network heterogeneities in the spread of STD sheds further light on the role of core groups in STD transmission dynamics. ${ }^{24}$ One feature of the spread of infection within a network is the rapid build up of correlations in the infection status of connected individuals-most infected individuals have infected sex partners, who have either transmitted the infection to them or acquired the infection from them. Such aggregation slows the spread of an epidemic by reducing the average number of susceptible partners per infected individual. ${ }^{24}$ Thus, within core groups the number of susceptibles will be depleted and STI spread will be curtailed if the size of the core group is not too large, the turnover rate (movement into and out of the core group) is small, the volume of sexual contacts between the core group and the periphery is limited, and, in the case of curable bacterial STI, the health system does not aggressively intervene to rapidly return infected individuals to susceptible status. During a recent outbreak of primary and secondary syphilis in Vancouver, British Columbia, the mass treatment intervention that was implemented resulted in increased incidence of primary and secondary syphilis, by returning infected people to susceptible status and thereby increasing the pool of susceptible individuals. ${ }^{25}$

Standardised and repeated measurement of quantifiable parameters related to core groups, such as the absolute and relative size (relative to the size of the total population) of core groups, changes in core group size, movement into and

Abbreviations: SW, sex worker. 
out of core groups, spatial movement of core groups, and the volume of sexual contact between core groups and the rest of the population may greatly enhance our understanding of the spread of STI in populations.

\section{Sexual mixing}

Earlier uses of the concept of core groups tended to be descriptive and theoretical, but in recent years STD researchers have focused increasingly on their quantifiable aspects. ${ }^{21}$ One analysis of sexual mixing between core and peripheral groups among African and white Americans suggested that the sizeable differential in STD rates between these two populations could be accounted for by their different rates of core to periphery sexual mixing. ${ }^{26}$ Ongoing work in Cotonou, Benin, explores qualitative and quantitative characteristics of behaviours and STI morbidity among female sex workers (SW) and their clients. ${ }^{27}{ }^{28}$ These investigations described the extent of variability in sex work and SW-client connections across local areas and over time. Rapid assessments in the Russian towns Saratov, Balakovo, and Engels suggested that such methodologies may allow estimation of some parameters related to sex work and SW-client contacts. ${ }^{29}$ An individual based simulation model was developed, using estimates from Saratov Oblast (the region), to explore how the number of client contacts an SW makes, whether clients repeatedly visit the same SW or many different ones, and the relative size of the SW and client populations all influence the establishment and endemic prevalence of gonorrhoea (a short duration infection) and herpes simplex virus-2 (HSV-2) (a longer duration infection). ${ }^{30}$ For both pathogens, infection was more likely to persist if clients visited many different SWs, regardless of variation in the frequency of such contacts. This scenario also resulted in a higher endemic prevalence in SW and client populations. The size of the SW population (relative to the total population) was most important in determining the overall prevalence of infection, with larger populations of SWs resulting in a higher overall prevalence.

These findings also point to the importance of turnover in and spatial movement of SWs in STI spread. Increases in turnover and spatial movement of SW populations may increase the numbers of specific SW-client links and perhaps influence STI spread in the same manner as larger SW populations. Monitoring sexual behaviour parameters among core groups must, therefore, include the measurement or estimation of spatial movement and turnover in SW populations. Spatial movement may cover different time periods, and may involve seasonal repeat migration or long term migration to another locale where the sex market may be more profitable. ${ }^{31}$ Movement of SWs may be closely related to their chronological and professional age and to the supply and demand conditions of the market. The average age of SWs, years in the profession, and average age at initiation into sex work may be helpful indicators of turnover. As with any other parameter, the distributions of these variables are even more informative than their measures of central tendency.

Clustering of STIs within populations means STI prevalence and incidence are high in some subpopulations and low in others. Mixing (and bridging) between members of high and low prevalence subpopulations may constitute risk factors for acquisition and transmission of STIs at the individual level and may facilitate spread at the population level. ${ }^{32}$ Sexual mixing between SWs and their clients is a classical example of such mixing. During the early 1980s, having sex with people from particular cities/countries was shown to be a risk factor for HIV infection, pointing to the importance of spatial measures of sexual mixing. Some studies of sexual mixing and bridge populations define these concepts in purely behavioural terms, with little reference to levels of STD prevalence and incidence. ${ }^{33-35}$ In the absence of STIs, risky sexual behaviours may not be associated with the acquisition of STDs. It is therefore important to include the biomedical indicators of STI in the definition of core groups, bridge populations, and sexual mixing. ${ }^{36}$ In a recent study of people infected with gonorrhoea and chlamydia in Seattle, Washington, $5.2 \%$ of respondents reported having had sex with people who lived outside their area of residence, over a period of three months. ${ }^{37}$ Based on data on sexual mixing/ bridging patterns among a general population sample of Seattle residents, $47.5 \%$ of those who had had one or more sex partners during their lifetime reported that at least one of their last two partners was not a Seattle resident. Among respondents who reported one or more concurrent sex partnership over the previous 12 months, $43.7 \%$ reported at least one sex partner who lived outside Seattle. ${ }^{38}$ Thus, in Seattle spatial bridging occurs considerably more frequently among members of the general population than it does among the infected population. Although spatial bridging is not in itself a high risk activity, sexual mixing (bridging) with residents of geographical areas where incidence/prevalence of STI is higher may constitute a high risk activity.

\section{THE ROLE OF SEXUAL BEHAVIOUR IN STI TRANSMISSION}

Untangling the role of behaviour in the transmission of HIV and other STIs has proved difficult. Observational studies that explore the relation between behavioural and biomedical outcomes often fail to show a strong relation between behaviours and acquisition of STI. ${ }^{39}$ Some behavioural intervention studies have also failed to show a strong relation between behaviours and STD acquisition. The association between behaviour and infection in observational studies is often either difficult to interpret or misleading.

\section{Misintrepretation due to context, study population, and partner's infection status}

Misinterpretation of the relation between sexual behaviour and STI transmission can occur in a number of ways. Firstly, both the risk and preventive behaviours of the respondent may not be fully considered and interpreted in the context of each other. For example, it is often assumed that condom use is protective: someone who uses condoms $50 \%$ of the time is assumed to be at a lower risk than someone who does not use them. Yet the non-user may be at lower risk, because they have, say, unprotected sex 10 times per month, while the $50 \%$ user has sex 30 times per month, of which 15 encounters are unprotected..$^{40}$

Secondly, associations between behaviour and infection differ by study population. Recent empirical evidence confirms that sexual risk behaviours vary by study population. A comparison of African-American women attending an STD clinic in North Carolina with African-American women in the surrounding community found statistically significant differences between the two populations with respect to marital status, employment status, income, number of sex partners, types of sex partners, whether or not the main partner had other sex partners, and whether or not the main partner had had an STD. ${ }^{41}$ Another study compared an STD clinic population to a general population sample obtained from a random digit dialling telephone survey and looked at factors associated with gonococcal infection. ${ }^{42}$ Three risk factors (age, black race, and whether or not the partner had spent a night in jail) emerged as important for gonorrhoeal infection in the STD clinic population. In the random digit dialling sample the above three risk factors were significant but five additional factors were also important. These included education at or above high school level, anal sex, 
history of any STD, having met partners in structured settings, and duration of relationship. These findings point to the importance of study population in determining findings related to sexual behaviour measurement and reinforce the argument that different study populations may be appropriate for the measurement of behaviours related to transmission probability, sexual exposure between infected and susceptible people, and duration of infectiousness.

Misinterpretation can also occur if the infection status of the partner is unknown, or not taken into account. ${ }^{43}{ }^{44}$ The risk of acquiring an STI depends on whether the partner is infected. Regardless of behaviour, infection cannot be acquired from uninfected partners. Thus, the risk in risky sexual behaviours such as unprotected intercourse or receptive anal intercourse without condoms is embedded in the infection status of the partner, which is not known except in the context of discordant partner studies. Moreover, behaviours are related to the infection status of the partner: people are more likely to have safe sex with risky partners and risky sex with safe partners. ${ }^{44}$ Statistical adjustment for such confounding necessitates knowledge of the infection status of each partner. More detailed information on sex partners may enhance our ability to assess partners' infection status. The numerous behavioural indicators used to reflect partners' infection probability, such as number of partners, duration and type of partnerships, concurrency, mixing (bridging) with high prevalence subpopulations, sex with drug users, sex with SWs, and so on, appear to be insufficient. In the context of intervention trials where the focus of sexual behaviour measurement is identification of risk factors for STI acquisition, inclusion of people whose partners are uninfected apparently creates a lack of precision in measuring associations of behaviours and STD risk and reduces the power of the study. ${ }^{44}$

\section{Interdependency and conditionality of sexual behaviours}

As data on sexual behaviour accumulate, the interdependencies among specific sexual behaviours and between epidemiological parameters and behaviours become increasingly clear. The examples are many: among gay men frequency of risk behaviour increases as their viral load decreases; similarly, men who have sex with men on highly active retroviral therapy may increase their high risk sexual activity and HIV infected people on highly active retroviral therapy may stop using condoms; among people infected with HSV-2 outbreaks lead to abstinence from sex; people who use condoms tend to have sex with higher risk sex partners; some men who have sex with men whose partners are HIV+ tend to avoid receptive anal intercourse; people who know they are at high risk of exposure to STIs (such as SWs) tend to get periodic check-ups; number of sex partners affects the frequency of intercourse with each partner; condom use affects sexual practices such that some people may refuse to have receptive anal intercourse in the absence of condoms. A classical example of these interdependencies is the positive correlation between condom use and STD; ${ }^{6}{ }^{40} 44$ a more recent complex example of multiple interdependencies is the tendency among men who have sex with men to engage in strategic positioning with or without condoms, and with or without ejaculation.

The interdependencies among behaviours that influence the spread of STI and between behaviours and infection necessitate that risk behaviours are conceptualised as mutually dependent, and measured as such. People apparently behave in ways which indicate that their sexual behaviours are patterned in "if" clauses. For example, they wear a condom if they or their partner is infected; they feel they can have many sex partners if they get frequently tested and know they are uninfected; they have receptive anal intercourse without a condom if they are infected or if they know their partner is uninfected. It is, therefore, important to interpret behavioural findings in the appropriate behavioural context, and identify and measure all relevant interrelated behaviours.

\section{Timing}

The timing of the measurement of sexual behaviour may affect the association between behaviour and infection. Many studies which measure sexual behaviour include, as respondents, people visiting STD clinics. People present at STD clinics are usually there because they have an STD or because they are concerned about a recent exposure to an STI; in other words their behaviour has been particularly risky in the recent past. If study participants are selected at a time when their behaviour has been recently particularly risky, sexual risk behaviours will tend to be overestimated. Moreover their behaviour in the following few months will be more like their usual behaviour and will be less risky-a phenomenon known as "regression to the mean". ${ }^{40}$ A regression to the mean would also be expected when measuring sexual behaviours of people accessing HIV counselling and testing services. At the population level, similar changes in behaviour are observed in response to HIV epidemics. As a result partly of the selective higher mortality of core group members, ${ }^{45}$ and partly of reductions in sexual risk behaviours of the survivors, many populations and subpopulations present less risky sexual behaviours following HIV epidemics. Behaviours of men who have sex with men in the United States, ${ }^{45}$ and of the general population in Uganda ${ }^{46} 47$ are well known examples of such changes.

The effects of timing may also complicate the measurement of relations between sexual behaviour and a number of other variables including infection status, other behavioural and biomedical risk factors, and societal and contextual determinants. Cross sectional studies often look for associations between infection rates and sexual behaviour by focusing on current sexual behaviour. ${ }^{48}$ Yet the behaviours responsible for current infection rates may have been engaged in 10-15 years earlier and may have changed following the emergence and spread of infection. Results of behavioural intervention trials conducted with STD clinic attendees as participants may be particularly vulnerable to the effects of timing on the measurement of sexual behaviour. ${ }^{40}$ Temporal changes in sexual behaviour have a differential effect on the measured associations between behaviours and specific STIs. Infections such as gonorrhoeal and chlamydial infection (short duration infections) are in general acquired as a result of recent sexual behaviours, whereas infections with HIV and HSV-2 (long duration infections) may be acquired through behaviours that took place decades earlier.

\section{Specific behaviours and specific STIs}

The interaction between the epidemiological properties of the sexually transmitted pathogen and the behaviours relevant to its spread often receives inadequate attention in the measurement of sexual behaviour. "The initial spread and long-term behaviour of any infectious disease are determined by both its epidemiological characteristics and the graph theoretical properties of the network-such as the average number of neighbours, degree of clustering and the path length between nodes." 24 An additional determinant of the initial spread and long term behaviour of curable bacterial STI, alongside those mentioned above, is the ability of healthcare systems to diagnose and treat the infected, sometimes with unexpected results. For example, mass 
treatment of core groups in Vancouver, British Columbia, in response to a primary and secondary syphilis epidemic, eventually led to increases in syphilis. ${ }^{25}$ More central to the task of measuring sexual behaviour, however, is the implicit assumption that the same sexual behaviours are equally important to the spread of all STIs. Recent mathematical modelling work suggests that the behaviours responsible for the ongoing transmission of HSV-2 and gonorrhoea may be different. ${ }^{49}$ For short lived, high transmission probability infections like gonorrhoea, the number of sex partners may be more important than the number of sex acts with each partner. For long duration, low transmission probability infections such as HSV-2, a moderate number of sex partners, with many acts with each partner, may maximise the number of infections generated by an infected individual. ${ }^{49}$ The importance of other variables describing sex partner networks may also differ according to the biology of infection. For example, concurrency of partnerships and short gaps between partnerships ${ }^{50}$ may be more important in the spread of high transmission probability, short duration infections, ${ }^{49}$ and relatively less important in the spread of low transmission probability, long duration infections.

These observations may help guide sexual behaviour measurement efforts in the future, and may help expand behavioural intervention repertoires. Such considerations may also in part account for some counterintuitive findings in STD epidemiology.

\section{Study design and STD transmission dynamics}

Epidemiological models suggest that in order to make valid estimates of the effect of behaviour on infection, it is important to differentiate between at least three distinct components of STD transmission dynamics: transmissibility of infection upon exposure between an infected and an uninfected person, likelihood of sexual exposure between infected and uninfected individuals, and duration of infection among infected people. Despite clear recognition of these three distinct components of STD transmission dynamics, studies of the relation between sexual behaviour and STD transmission do not, in general, adequately differentiate between them.

Different study designs may be appropriate for the measurement of sexual behaviours relevant to each of the three components. For example, behaviours related to transmissibility, such as condom use, can be evaluated effectively only in discordant partners. ${ }^{51-53}$ Behaviours related to sexual exposure between infected and uninfected individuals may be effectively studied among partnered and nonpartnered people. Populations with very high and very low levels of prevalence/incidence may not be appropriate for the study of behaviours related to sexual exposure between infected and uninfected. ${ }^{39}$ Finally, behaviours related to duration of infectiousness including healthcare seeking, testing, and therapy compliance behaviours, would best be studied in infected or very high risk populations.

The particular STI under consideration may also have important implications for the choice of study population. Levels of prevalence and incidence (as mentioned above), transmission probability and duration of infectiousness vary with specific STIs.

\section{CONCLUSION}

The measurement of sexual behaviour serves a number of distinct purposes including assessment and temporal monitoring of risk behaviours in populations and the assessment of the role of sexual behaviour in the acquisition and transmission of specific STI. Evidence to date makes it clear that the purpose for which the measurement is undertaken is vitally important in defining the sexual behaviours to be measured, the populations to be studied, the time periods to be covered, and the contextual parameters to be considered. The specific purpose of the measurement may, for example, determine whether sexual behaviour is conceptualised at the individual or the population level. Sexual behaviour measurement is a complex and complicated business and needs to be undertaken with a precise grasp of the purposes of the endeavour.

\section{ACKNOWLEDGEMENTS}

The author would like to acknowledge Patricia Jackson for her outstanding support in the preparation of this article.

Correspondence to: Dr S O Aral, Associate Director for Science,

Division of STD Prevention, Centers for Disease Control and Prevention, 600 Clifton Road, Mailstop E02, Atlanta, GA 30333, USA; soal@cdc.gov

\section{REFERENCES}

1 Laumann EO, et al. The social organization of sexuality: sexual practices in the United States. Chicago: University of Chicago Press, 1994.

2 Johnson AM, Mercer CH, Erens B, et al. Sexual behaviour in Britain: partnerships, practices, and HIV risk behaviours. Lancet 2001;358:1835-42.

3 Ku L, St Louis M, Farshy C, et al. Risk behaviors, medical care, and chlamydial infection among young men in the United States. Am J Public Health 2002:92: 11 140-43.

4 Foxman B, Aral SO, Holmes KK. Interrelationships among douching practices, risky sexual practices, and history of self-reported sexually transmitted diseases in an urban population. Sex Transm Dis 1998;25:90-99.

5 Rogers SM, Miller HG, Miller WC, et al. NAAT-identified and self-reported gonorrhea and chlamydial infections: different at-risk population subgroups? Sex Transm Dis 2002;29:588-96.

6 Zenilman JM, Weisman CS, Rompalo AM, et al. Condom use to prevent incident STDs: the validity of self-reported condom use. Sex Transm Dis 1995;22:15-21.

7 Weir SS, Feldblum PJ. Condom use to prevent incident STDs. Sex Transm Dis 1996;23:76-77; discussion 79-82.

8 Turner CF, Miller HG. Zenilman's anomaly reconsidered: fallible reports, ceteris paribus, and other hypotheses. Sex Transm Dis 1997;24:522-27.

9 Fishbein $M$, Jarvis B. Failure to find a behavioral surrogate for STD incidencewhat does it really mean? Sex Transm Dis 2000;27:452-55.

10 Kelly JA, Murphy DA, Washington CD, et al. The effects of HIV/AIDS intervention groups for high-risk women in urban clinics. Am J Public Health 1994;84:1918-22.

11 DiClemente RJ, Wingood GM. A randomized controlled trial of an HIV sexual risk-reduction intervention for young African-American women. JAMA 1995;274:1271-6.

12 St Lawrence JS, Brasfield TL, Jefferson KW, et al. Cognitive-behavioral intervention to reduce African-American adolescents' risk for HIV infection. J Consult Clin Psychol 1995;63:221-37.

13 Jemmott JB, Jemmott LS, Fong GT. Abstinence and safer sex HIV riskreduction interventions for African American adolescents: a randomized controlled trial. JAMA 1998;279:1529-36.

14 Shain RN, Piper JM, Newton ER, et al. A randomized controlled trial of a behavioral intervention to prevent sexually transmitted disease among minority women. N Engl J Med 1999;340:93-100.

$15 \mathrm{Kamb}$ ML, Fishbein M, Douglas JM, et al. Efficacy of risk-reduction counseling to prevent human immunodeficiency virus and sexually transmitted diseases: a randomized controlled trial. Project RESPECT Study Group. JAMA 1998;280:1161-7.

16 Pisani E, Garnett GP, Brown T, et al. Back to basics in HIV prevention: focus on exposure. BMJ 2003;326:1384-7.

17 Liljeros F, Edling CR, Amaral LAN. Sexual networks: implications for the transmission of sexually transmitted infections. Microbes Infect 2003;5: 189-96.

18 Liljeros F, Edling CR, Amaral LAN, et al. The web of human sexual contacts. Nature 2001;411:907-8.

19 Schneeberger A, Mercer CM, Gregson SAJ, et al. Scale free networks and sexually transmitted diseases: a description of observed patterns of sexual contacts in the UK and Zimbabwe. Presented at the 15th Biennial Congress of the International Society for Sexually Transmitted Diseases Research (ISSTDR). Ottawa, Canada, July 2003.

20 Foxman B, Aral SO, Holmes KK. Heterosexual repertoire is associated with same-sex experience. Sex Transm Dis 1998;25:232-36.

21 Thomas JC, Tucker MJ. The development and use of the concept of a sexually transmitted disease core. J Infect Dis 1996;174(Suppl 2):S134-43.

22 Steen R, Vuylsteke B, DeCoito T, et al. Evidence of declining STD prevalence in a South African mining community following core group intervention. Sex Transm Dis 2000;1:1-8.

23 Van Dam CJ, Holmes KK. STD prevention: effectively reaching the core and a bridge population with a four-component intervention (editorial). Sex Transm Dis 2000;27:9-11.

24 Eames KTD, Keeling MJ. Modeling dynamic and network heterogeneities in the spread of sexually transmitted diseases. PNAS 2002;99:13330-35. 
25 Rekart $M$, Wong $T$, Wong $E$, et al. Evaluation of British Columbia's syphilis mass treatment intervention. Abstract presented at the 15th Biennial Congres of the International Society for Sexually Transmitted Diseases Research (ISSTDR). Ottawa, Canada, July 2003.

26 Laumann EO, Youm Y. Racial-ethnic group differences in the prevalence of sexually transmitted diseases in the United States: a network explanation. Sex Transm Dis 1999;26:250-61

27 Lowndes CM, Alary M, Labbe AC, et al. Male clients of female sex workers in Cotonou, Benin (West Africa): contribution to the HIV epidemic and effect of targeted interventions. Abstract presented at the 15th Biennial Congress of the International Society for Sexually Transmitted Diseases Research (ISSTDR). OHtawa, Canada, July 2003.

28 Alary M, Lowndes CM, Labbe AC, et al. Interventions aimed at sex workers in Cotonou, Benin (West Africa): ongoing decline of STDs over a decade. Abstract presented at the 15th Biennial Congress of the International Society for Sexually Transmitted Diseases Research (ISSTDR). Ottawa, Canada, July 2003

29 Aral SO, St Lawrence JS. The ecology of sex work and drug use in Saratov Oblast, Russia. Sex Transm Dis 2002;29:798-805.

30 Ghani AC, Aral SO. Patterns of sex worker-client contacts and their implication for the persistence of STIs. J Infect Dis 2004 (in press).

31 Blanchard J, Ramesh R, Orchard T, et al. Sex work in India: patterns embedded in societal tradition. Abstract presented at the 15th Biennia Congress of the International Society for Sexually Transmitted Diseases Research (ISSTDR). OHtawa, Canada, July 2003.

32 Aral SO, Hughes JP, Stoner B, et al. Sexual mixing patterns in the spread of gonococcal and chlamydial infections. Am J Public Health 1999;89:825-33.

33 Morris M, Podhisita C, Wawer MJ, et al. Bridge populations in the spread of HIV/AIDS in Thailand. AIDS 1996;10:1265-71.

34 Havanon N, Bennett A, Kondel J. Sexual networking in provincial Thailand. Stud Fam Plann 1993;24:1-17

35 Gorbach PM, Sopheab H, Phalla T, et al. Sexual bridging by Cambodian men: potential importance for general population spread of STD/HIV epidemics. Sex Transm Dis 2000;27:320-6.

36 Aral SO. Behavioral aspects of sexually transmitted diseases: core groups and bridge populations. Sex Transm Dis 2000;27:327-8

37 Kerani RP, Golden MR, Whittington WLH, et al. Spatial bridges for the importation of gonorrhea and chlamydial infection. Sex Transm Dis 2003;30:742-9.

38 Aral SO, Foxman B. Spatial mixing and bridging: risk factors for what? Sex Transm Dis 2003;30:750-1.

39 Aral SO, Peterman TA. Editorial: A stratified approach to untangling the behavioral/biomedical outcomes conundrum. Sex Transm Dis 2002:29:530-2.
40 Peterman TA. Can we measure STD risk behavior or STD as surrogates for HIV risk? Paper presented at International Congress of Sexually Transmitted Infections (ISSTDR/IUSTI) 2001, Berlin. BundesgesundheitsblGesundheitsforsch-Gesundheitsschutz 2002;45:286-90.

41 Howards PP, Thomas JC, Earp JA. Do clinic-based STD data reflect community patterns? Int J STD AIDS 2002;13:775-80.

42 Manhart LE, Aral SO, Holmes KK, et al. Influence of study population on the identification of risk factors for sexually transmitted diseases using a casecontrol design: the example of gonorrhea. Am J Epidemiol 2004; 160:393-402.

43 Shain RN, Perdue ST, Piper JM, et al. Behaviors changed by intervention are associated with reduced STD recurrence: the importance of context in measurement. Sex Transm Dis 2002;29:520-9.

44 Peterman TA Lin LS, Newman DR, et al. Does measured behavior reflect STD risk? An analysis of data from a randomized controlled behavioral intervention study. Sex Transm Dis 2000;27:446-51.

45 Chesson HW, Dee TS, Aral SO. AIDS mortality may have contributed to the decline in syphilis rates in the United States in the 1990's. Sex Transm Dis 2003:30:419-24.

46 Orroth AK. Investigations of the proportion of HIV infections attributable to sexually transmitted diseases in sub-Saharan Africa based on data from the Mwanza and Rakai trials. PhD thesis, Faculty of Medicine, University of London, 2003.

47 Korenromp EL, Bakker R, de Vlas SJ, et al. HIV dynamics and behaviour change as determinants of the impact of sexually transmitted disease treatment on HIV transmission in the context of the Rakai trial. AIDS 2002; 16:2209-18

48 Auvert B, Buve A, Ferry B, et al. Ecological and individual level analysis of risk factors for HIV infection in four urban populations in sub-Saharan Africa with different levels of HIV infection. AIDS 2001;15(Suppl 4):S15-S30.

49 Garnett GP. The geographical and temporal evolution of sexually transmitted disease epidemics. Sex Transm Infect 2002;78(Suppl 1):i14-i19.

50 Kraut-Becher JR, Aral SO. Gap length: an important factor in sexually transmitted disease transmission. Sex Transm Dis 2003;30:221-5.

51 Fenton KA, Johnson AM, McManus S, et al. Measuring sexual behaviour: methodological challenges in survey research. Sex Transm Inf 2001;77:84-92.

52 Aral SO, Peterman TA. STD diagnosis and treatment as an HIV prevention strategy. In: O'Leary A, ed. Beyond condoms: alternative approaches to HIV prevention. New York: Kluwer Academic/Plenum Publishers, 2002:77-90.

53 Warner L, Newman D, Peterman TA, et al. Studying condom effectiveness for sexually transmitted disease prevention: the importance of knowing partner infection status (abstract B9B). Presented at the 2002 National STD Prevention Conference, San Diego, California, 4-7 March 2002. 